\title{
INDEX TO SCIENTIFIC NAMES
}

Acacia catechu 632

Acer ginnala 656

Aceraceae 656

Acorus calamus 739

Acorus tatarinowii 740

Adonis amurensis 620

Aeginetia indica 714

Agkistrodon acutus 777

Aleurites moluccana 645

Allium cepa 731

Alnus japonica 605

Alocasia cucullata 741

Alum 791

Amarantaceae 616

Amaryllidaceae 735

Amomum villosum 745

Anagallis arvensis 685

Anas domestica 778

Anatidae 778

Anguilla japonica 763

Anguillidae 763

Annonaceae 618

Apocynaceae 696-699

Aquillaria agallocha 667

Araceae 739-743

Araliaceae 679

Arca subcrenata 749

Arcidae 749

Artemisia argyi 719

Ascelpiadaceae 700

Asclepias curassavica 700

Aspidistra elatior 732

Aster scaber 720

Atropa belladonna 710

Aucklandia lappa 721

Baeckea frutescens 676

Bambusa tuldoides 737

Betula japonica 605

Betulaceae 605

Bidens tripartita 722

Bignoniaceae

Blumea balsamifera 723

Bolbostemma paniculatum

671

Bombycidae 756

Bombyx mori 756

Bos taurus domesticus 785

Boschniakia rossica 715

Boswellia carterii 652
Bovidae

Brassica alba 627

Bruseraceae 653-654

Bubalus bubalis 786

Bufo bufo gargarizans 770

Bufonidae 770

Bungarus multicinctus

multicinctus 776

Bupleurum falcatum 680

Burseraceae 652

Buthidae 754

Buthus martensii Karsch 754

Callicarpa pedunculata

703

Callorhinus ursinus 782

Campanulaceae 717-718

Canarium album 653

Carassius auratus 765

Carassius carrassius 765

Carcharhinidae 762

Carcharhinus menisorrah 762

Caryophyllaceae 611-613

Cassia angustifolia 633

Catharanthus roseus 696

Cayratia japonica 660

Cedrela sinensis 655

Celastraceae 658-659

Ceratotherium simum 784

Cerbera manghas 697

Cerbera odollam 697

Cervidae 788-789

Cervus elaphus 788

Cervus nippon 788

Chenopodiaceae 614-615

Chenopodium ambrosioides

614

Chinemys reevesii 771

Chrysanthemum

cinerariaefolium 724

Cicadidae 757-758

Cissus japonica 660

Citrus junos 648

Clerodendrum inerme 704

Cnidium monnieri 681

Cobitidae 764

Collocalia esculenta 780

Collocalidae 780
Colocasia antiquorum 742

Colubridae 775

Combretaceae 677-678

Commiphora molmol 654

Commiphora myrrha 654

Compositae 719-730

Convolvulaceae 701-702

Corchorus acutangulus 663

Corydiidae 759

Crassostrea gigas 750

Cruciferae 627

Cryptotympana atrata 757

Cryptotympana pustulata

758

Cucumis melo var. makuwa

672

Cucumis sativus 673

Cucurbitaceae 671-674

Cudrania cochinchinensis

606

Curcuma zedoaria 746

Cyprinidae 765-766

Cyprinus carpio 766

Dahlia variabilis 725

Dalbergia odorifera 634

Daucus carota 682

Dendrobium moniliforme

747

Desmodium styracifolium

635

Desmos chinensis 618

Dicerorhinus sumatrensis

784

Diceros bicornis 784

Dimocarpus longan 657.

Diospyros lotus 687

Diploclisia glaucescens 623

Dipterocarpaceae 626

Doellingera scaber 720

Dolichos lablab 636

Drymaria cordata 611

Drymaria diandra 611

Drynaria fortunei 602

Dryobalanops aromatica

626

Dryobalanops Camphora

626

Duranta repens 705 
Ebenaceae 687

Elapidae 776

Entada phaseoloides 637

Epidendrum moniliforme

747

Epsomite 792

Equidae 783

Equus asinus 783

Ericaceae 684

Erigeron canadensis 726

Erodium stephanianum 642

Erycike obtusfolia 701

Eucalyptus globulus 677

Euonymus japonica 658

Euonymus sieboldianus 659

Euphorbia sieboldiana 646

Euphorbiaceae 645-647

Euphoria longan 657

Eupolyphaga sinensis 759

Fagopyrum cymosum 609

Ficus religiosa 607

Firmiana platanifolia 665

Firmiana simplex 665

Fragaria chiloensis var.

ananassa 629

Fritillaria cirrhosa 733

Gallus gallus domesticus

779

Gekko gecko 773

Gekko swinhonis 774

Gekkonidae 773-774

Gentiana macrophylla 694

Gentianaceae 694-695

Geraniaceae 642

Gladiolus gandovensis 736

Glechoma hederacea 706

Glechoma hederacea var.

grandis 706

Gnaphalium affine 727

Gnaphalium multiceps 727

Gnetaceae 604

Gnetum parvifolium 604

Gomphrena globosa 616

Gramineae 737-738

Gypsum Fibrosum 793

Haematite 794

Haliotidae 748

Haliotis diversicolor 748

Hamamelidaceae 628

Hibiscus manihot 664
Hippocampus histrix 767

Hippocampus japonicus 767

Hippocampus kelloggi 767

Hippocampus kuda 767

Hippocampus trimaculatus

767

Hirudinidae 753

Hirudo nipponia 753

Inula linariifolia 728

Ipomoea brasiliensis 702

Ipomoea pes-caprae 702

Iridaceae 736

Jasminum sambac 690

\section{Kieserite 792}

Labiatae 706-709

Lactuca sativa 729

Lactuca scariola var. sativa 729

Lagerstroemia indica 675

Lardizabalaceae 621-622

Lauraceae 619

Laurus nobilis 619

Leguminosae 632-640

Ligustrum purpurascens

691

Ligustrum robustum 692

Liliaceae 731-734

Lobelia chinensis 717

Loranthaceae 608

Loropetalum chinensis 628

Lythraceae 675

Maclura cochinchinensis

606

Magnolia coco 617

Magnoliaceae 617

Malvaccac 664

Mantidae 760

Masakia japonica 658

Megascolecidae 752

Meliaceae 655

Menispermaceae 623-624

Menyanthus trifoliata 695

Mirabilite 795

Misgurnus anguillicaudatus

764

Moraceae 606-607

Moschus moschiferus 789

Mucuna birdwoodiana 638
Musa basjoo 744

Musaceae 744

Mustelus griseus 762

Myrtaceae 676-678

Oenanthe javanica 683

Oenanthe stolonifera 683

Oleaceae 690-693

Ophicephalidae 769

Ophicephalus argus 769

Orchidaceae 747

Orobanchaceae 714-715

Oroxylum indicum 713

Osmanthus fragrans var.

aurantiacus 693

Osteridae 750

Ostrea gigas 750

Oxalidaceae 641

Oxalis corniculata 641

Panax quinquefolius 679

Panicum viride 738

Paratenodera sinensis 760

Passiflora foetida 669

Passifloraceae 669

Phasianidae 779

Pheretima aspergillum 752

Phoca groenlandica 782

Phoca vitulina 782

Phocidae 782

Physeter catodon 781

Physeter macrophalus 781

Physeteridae 781

Pinaceae 603

Pinus koraiensis 603

Pogostemon cablin 707

Polycarpaea corymbosa

612

Polygonaceae 609-610

Polygonatum chinense 734

Polygonatum sibiricum 734

Polypodiaceae 602

Pratia nummularia 718

Primula sieboldii 686

Primulaceae 685-686

Prunus tomentosa 630

Pseudo-acacia odorata 640

Psidium guajava 678

Ranunculaceac 620

Rauwolfia serpentina 698-

699

Realgar 769 
Rhincodon typus 762

Rhincodotidae 762

Rhinoceros sondaicus 784

Rhinoceros unicornis 784

Rhinocerotidae 784

Rhododendron

mucronulatum 684

Robinia pseudo-acacia 640

Rosaceae 629-631

Rumex acetosa 610

Ruta graveolens 649

Rutaceae 648-651

Saiga tatarica 787

Salvia officinalis 708

Sapindaceae 657

Sargassaceae 601

Sargassum pallidum 601

Sargentodoxa cuneata 621

Saururaceae 625

Saururus chinensis 625

Saussrea lappa 721

Scolopendra subspinipes 755

Scolopendridae 755

Scrophularia ningpoensis

712

Scrophulariaceae 712

Scutellaria barbata 709

Securinega suffruticosa 647

Sepiella maindroni 751

Sepia esculenta 751

Sepiidae 751

Setaria viridis 738

Sinapis alba 627

Smithsonite with Dolomite 797
Solanaceae 710-711

Solanum tuberosum 711

Solenognathus hardwick

768

Spinacia oleracea 615

Spiraea prunifolia var.

simpliciflora 631

Stalactit 798

Stauntonia chinensis 622

Stephania japonica 624

Sterculia lychnophora 666

Sterculiaceae 665-666

Stichopodidae 761,782

Stichopus japonicus 782

Styracaceae 688

Styrax japonica 688

Styrax tonkinensis 689

Stytacaceae 689

Suidae 790

Sulfur 799

Sus scrofa domesica 790

Syngnathidae 767-768

Syngnathoides biaculeatus

768

Syngnathus acus 768

Talc 800

Tamaricaceae 670

Tamarix chinensis 670

Tenodera sinensis 760

Testudinidae 771

Thymelaeaceae 667

Tiliaceae 663

Toona sinensis 655

Triakidae 762

Tribulus terrestris 644

Trichosanthes anguina 674
Trichosanthes cucumerina

var. anguina 674

Trifolium repense 639

Trionychidae 772

Trionyx sinensis 772

Tropaeolaceae 643

Tropaeolum majus 643

Typhonium giganteum 743

Umbelliferae 680-683

Vaccaria segetalis 613

Valeriana officinalis 716

Valerianaceac 716

Verbenaceae 703-705

Viola yedoensis 668

Violaceae 668

Viperidae 777

Viscum coloratum 608

Vitaceae 660-662

Vitis amurensis 661

Vitis vinifera 662

Vladimiria soulieri 730

Whitmania acranulata 753

Whitmania pigra 753

Zanthoxylum ailanthoides 650

Zanthoxylum schinifolium

651

Zaocys dhumnades 775

Zephyranthus candida 735

Zingiberaceae 745-756

Zygophyllaceae 644 


\section{中文索引（繁體字、简体字、日字）}

1 劃

宂一年蓬 (一年蓬) 726

\section{㕂公滕 701}

二葉紅薯（…叶红薯、二葉紅著） 702

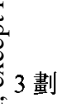

4 劃

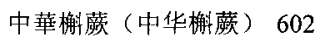

天䒾麥 (天芥麦, 天蓄麥) 609

王不留行 613

月桂 619

月桂子 619

牛藤 622

牛奶墏草 (牛奶浆草, 牛乳墏草) 646

水芹 683

水蛭 753

水牛 786

水牛角 786

日本絡石 (日本络石, 日本絡石) 699 木蝴蝶 713

木香 721

毛蚶 749

瓦楞子 749

少棘巨蜈蚣 (少棘巨蜈蚣, 少棘巨蜈

蚣） 755

巨斧螳蛨 760

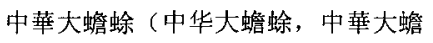

蜍） 770

五步蛇 777

牛 785

牛黄 785

5 劃

白鼓釷（白鼓钉） 612

白芥 627

白芥子 627

白扁豆 (白府豆, 白扁豆) 636
白花油麻藤 638

白車軸草 (白车轴草, 白車軸草) 639 白牡 659

白花映山紅（白花映山红） 684

白棠子樹（白棠子树） 703

白附子 743

白䱒 (白鲍) 748

白鴨肉 (白鸭肉, 白鴨肉) 778

白箬（白现） 791

白花樹 (白花树, 白花樹) 689

石栗 793

石菖蒲 740

石斛(石㓩) 747

石決明 (石决明, 石決明) 748

石旁 793

石遂 646

甘畫貝母（甘肃贝母） 733

瓜蒂 672

四念癀（四念癀, 四念癀） 685

半枝蓮 (半枝莲, 半枝蓮) 709

半邊蓮 (半边莲, 半辺蓮) 717

玄参 712

艾 719

艾葉 (艾叶, 艾葉) 719

玉策 (玉帘，玉策） 735

地龍（地龙, 地龍） 752

代褚石 794

\author{
6 劃 \\ 羊棲菜 (羊栖菜, 羊栖菜) 601 \\ 羊鮑（羊鲍） 748
}


印度菩提樹皮（印度菩提树皮, 印度 菩提樹皮） 607

冰涼花 (冰凉花, 水涼花) 620

造冰片（冰片，水片） 626

尖葉番潟 (尖叶番泻) 633

尖尾草 741

老制草 (老鹳草, 老鸛草) 642

西河柳 670

679

安息香樹 (安息香树) 689

安息香 (安息香) 689

粗壯女貞（粗壮女贞） $691 ， 692$

粗壮秦芜 694

亞洲絡石（亚洲络石,亞洲絡石）699

灰毛川木香（灰毛川木香，灰毛川木

香) 730

庅腰金絲燕 (灰腰金丝燕) 780

多花黄精 734

竹茹 737 劃

耳鮑（耳鲍） 748

全蠍（全蝎，全蠍） 754

地鳘(地鳖，地紫) 759

芒硝 795

ले 7 劃

ถิ

赤楊（赤杨，赫楊） 605

芥 627

芥子 627

旱金蓮（旱金莲，旱金蓮） 643

旱蓮花(旱莲花, 旱蓮花) 643

芸香 649

花椒 651

任旗参 679
乳香樹 (乳香树, 乳香樹) 652

乳香 652

沒藥樹（没药树，沒薬樹） 654

沒藥（没药, 沒薬） 654

沈香 (沉香, 沈香) 667

芹花 683

迎紅杜鵑（迎红杜鹃, 迎紅杜鵑） 684

君遷子（君迁子，君遷子） 687

杜虹花 703

假連䞨（假连趣） 705

肝風草（肝风草, 肝風草） 735

芭蕉 744

芭蕉頭（芭蕉头，芭蕉頭） 744

芭蕉葉 (芭蕉叶, 芭蕉葉) 744

陽春砂（阳春砂） 745

近江牡䗶 (近江牡蚛, 近江牡蚛) 750

牡蠣（牡蚛，牡蚛） 750

松子 603

夜合花 617

兒茶（儿茶，児茶） 632

降香檀 634

降香 634

刺槐 640

酢漿草（酢浆草，酢漿草） 641

青椒 651

青果 653

青杆竹 737

莱莉 690

茉莉花 690

苦丁茶 691,692

苦郎樹（苦郎树, 苦郎樹) 704
金木㺟 693

金沸花 728

金錢白花蛇（金钱白花蛇，金錢白花

蛇） 776

金絲燕 (金丝燕，金系燕) 780

長春花（长春花，長春花） 696

長牡蠴(长牡螈，長牡蚛) 750

東風菜（东风菜，東風菜） 720

狗尾草（狗尾草，犬尾草） 738

參環毛蚓 (参环毛蚓, 參環毛蚓) 752

東亞針蠍（东亚钳蝎，東亞鉗蠍） 754

泥鰍（泥鳅，泥鲰） 764

阿胶 783

林廦 789

明䙪（明矾） 791

9 劃

骨碎補 (骨·碎补, 骨砕補)

穿破石 606

星色草 612

扁豆（扁豆, 扁豆) 636

柚子 648

香椿 655

茶條椷(茶条械) 656

烏敏莓（鸟蔹莓，烏䓵莓） 660

胖大海（胖大海, 胖大海） 666

南五味子 675

南鶴禹(南鹤䖝，南鶴禹) 682

柿 687

馬尾松（馬尾松） 603

馬利筋(马利筋, 馬利筋) 700

馬鞍藤（马鞍藤） 702

馬黁（马鹿） 789 


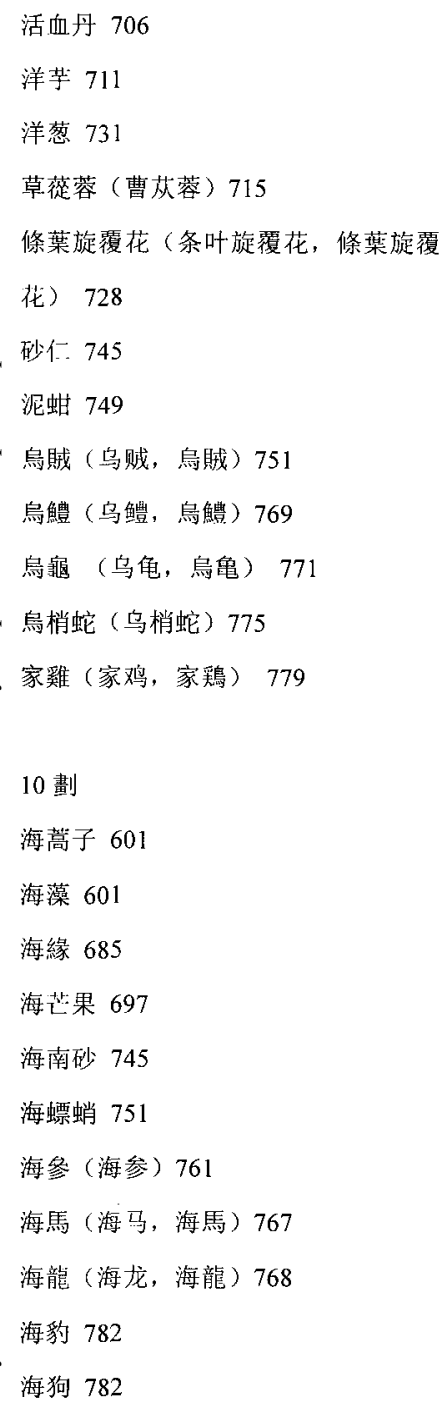

柴胡 680

桂花 693

秦芜 694

連錢草（连钱草，連錢草） 706

狼把草 722

狼把草根 722

除虫料 724

浙貝母（浙少母，浙貝母） 733

唐葛蒲 736

莪术 746

細菜石斛 (细蓄不斛) 747

威廉環毛蚓（威廉环毛蚓） 752

家薙（家虫） 756

桑蝶蛸 760

無蹼壁虎（乌䠊譬虎） 744

11 劃

紅松（红松，紅松） 603

買麻藤 (买麻藤) 604

菩提樹 (萻提树, 菩提樹) 607

野蓄麥 (野葬麦, 野䒾麥) 609

野黄麻 663

野胡蘿萄(野胡萝卜) 682

野茉莉 688

野㼋 714

野㘶 742

玟藍菜 (麦蓝莱, 麥藍莱) 613

菠菜 615

甜瓜 672

甜瓜葉(甜瓜叶, 甜瓜葉) 672

甜瓜子 672

蛇瓜 674

蛇床 681
蛇床子 681

蛇根木 698

旋覆花（旋覆花，旋覆化） 728

蓠芦 (莴芭) 729

茼芦子（莴草子） 729

梭砂貝母（梭砂贝母） 733

淡竹 737

通俗環毛蚛（通俗坏毛蚓，通俗環毛 蚓) 752

魚翅 (鱼翅, 魚翅) 762

鹿 788

琵茸: 788

鹿角 788

鹿尾 788

鹿筋 788

鹿胎 788

鹿蜼 788

鹿角籍 788

鹿角胶 788

猪（猪，豚) 790

猪膽（猪胆，豚胆） 790

㸱牛兒苗 (诫牛儿苗, 掝牛児苗) 642

12 劃

假鹰爪 618

假黄麻 663

荅白科鈎豐（苍白科钩风） 623

番瀉葉 (番泻叶,番瀉葉) 633

過崗龍（过岗龙） 637

鈎腺大戟 (钩腺大戟，鈎腺大戟) 646

葉底珠 (叶底珠, 葉底珠) 647

絲棉木 (丝棉木, 系棉木) 659

葡萄 662 
葡萄根 662

葡萄藤 662

葡萄滕葉(葡萄膯叶) 662

黃蜀葵（黄蜀葵，黃蜀蕟） 664

黄蜀葵根（黄蜀葵根，黄蜀葵根） 664

梧桐 665

梧桐皮 665

梧桐白皮 665

梧桐子 665

黄瓜(共瓜, 胡瓜) 673

黄瓜藤 (黄瓜藤, 胡瓜藤) 673

黃瓜花 (黄瓜仳, 湖瓜花) 673

黃瓜葉(黄瓜叶, 胡瓜葉) 673

黄精 734

崗松 (岗松, 岗松) 676

番石榴 678

番石榴葉 (番不榴叶, 番石榴菜) 678

麻花秦芜 694

黑水葡萄 661

黑虾: 757,758

黑眶䇾蜍 770

蛤蚧 773

單色金絲燕 (单色金丝燕) 780

犀牛 784

犀角 784

羚羊角(羚羊角, 羚羊角) 787

雄黄 796

硫黄 799

13 劃

跻槷 644

椿白度 655

椿葉(椿叶, 椿葉) 655
紫花地丁 668

紫薇 675

紫荆 675

紫荆皮 675

紫薇叶 675

紫薇化 675

紫薇根 675

紫荎女貞（紫茎女贞）691，692

紫珠 703

睡菜 695

睡菜葉（睡菜叶, 睡菜葉) 695

蓮生桂子花（莲生桂子花, 蓮生桂子

花） 700

鼠曲草 727

暗紫貝姆（暗紫贝母） 733

滇黄精 734

搜山黄 736

蓬莪术 746

滑石（滑石，滑石） 800

\section{4 劃}

构棘 606

檞䓲 602

判寄生 608

酸模 610

龍腦香 (龙脑香, 龍脳香) 626

龍眼 (龙眼, 龍眼) 657

龍眼肉 (龙眼肉, 龍眼肉) 657

龍眼根 (龙眼根, 龍眼根) 657

龍眼葉 (龙眼叶, 龍眼葉) 657

龍眼核 (龙眼核, 龍眼核) 657

龍珠果 (龙珠果, 龍珠果) 669

龍涎香（龙涎香, 龍涎香) 781
廣金錢草 (广金钱草，広金錢草） 635

鯽魚 (鲫鱼) 765

鯉魚 (鲤鱼, 鯉魚) 766

鯉魚膽（鲤鱼胆, 鯉魚胆) 766

潟利鹽 (泻利盐, 潟利嫶) 792

翠藍草 (斐蓝草, 章監草) 686

絡石(络石, 絡石) 699

絡石藤（络不藤, 絡石滕) 699

絡石果 (络不果, 絡石果) 699

銅錪玉帶草（铜锤玉带草） 718

蜘蛛抱蛋 732

綠殸砂 (绿壳砂) 745

雜色鮑（杂色鲍, 雑仙鮑） 748

魁蚶 749

15 劃

橙子 648

橙子皮 648

㯪葉花椒 (㯪叶花椒, 㯪葉花树) 650

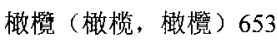

調經草 (调经草, 調経草) 658

廣東人參 (广东人参, 层東人参) 679

廣霍香 ( ’”藿香, 広蕉香) 707

酦紋盤鮑（坡纹盘鲍，策紋盤鮑）748

標杆花 (标杆花) 736

澳洲鮑（澳洲鲍） 748

螞蟥（蚂蟥） 753

蜈蚣（蜈蚣，蜈蚣）755

僵蝉(僵虫) 756

鴨 (鸭, 鴨) 778

鴨血（鸭血，鴨血） 778

赫石 794 
16 劃

藏葛蒲(藏葛蒲, 藏葛蒲) 739

獨角蓮( 独角莲, 独角蓮) 743

壁虎（壁虎，壁虎） 774

燕窩 (燕窝, 燕窝) 780

17 劃

檉柳 (柽柳) 670

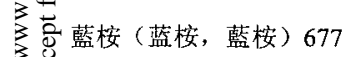

櫛盲環毛蚓 (栉盲环毛蚓) 752

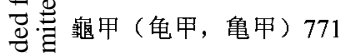

賽加羚羊（赛甲羚羊，賽加羚羊) 787

웅

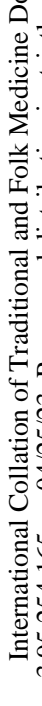

18 劃

顛茄（颠茄，顛茄） 710

顛茄草 (颠茄草, 顛茄草) 710

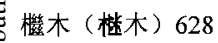

乷魚 (㸺鱼, 鯊魚) 762

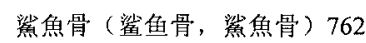

19 劃

蟬蛻（蝉蜕, 蝉蛻) 757,758

蟾酥（蟾酥，蟾酥) 770

鱉（鳖，鱉） 772

鱉甲（鳘甲，鰵甲) 772

䪊蛇 (蒴蛇) 777

ถ 20 劃

雞爪風（鸡爪风）618

雞内金（鸡内金，雞内金）779

爐甘石（炉甘石，炉甘石） 797

鍾乳石(钟乳石) 798

21 劃

櫻草 (樱草, 桜草) 686
櫻草根 (櫻草根, 桜草根) 686

鹰 789

麿香 789

22 劃

維芙木 (夢芙木, 蘿芙木) 698

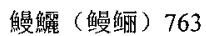

23 劃

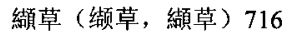

篦砂（幂沙） 756

24 劃

鱧魚（鳢鱼） 769

25 劃

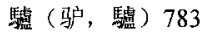

笽皮膠 (马户皮胶, 驉皮胶) 783

28 劃

查仁 (郁李仁) 630

李根 (郁李根) 63 


\section{INDEX TO LOCAL DRUG NAMES}

A-chul 746

Aeng-ch-geun 686

Aeng-cho 686

Aeng-do-na-mu 630

Ae-yeop 719

A-gga-si-na-mu 640

Ahiru 778

Ai 719

Ai-ye 719

Akyo 783

Amagasahebi 776

Amerika-ninjin 679

Amigasayuri 733

An-sik-hyang 689

Ansokuko 689

An-xi-xiang 689

An-zi-bei-mu 733

Aogiri 665

Ao-zhou-bao 748

Ap-hyul 778

Asenyaku 632

Asenyakunoki 632

A-seon-yak 632

Ba-da-pyo-beom 782

Baek-ap-yuk 778

Baek-gae-ja 627

Baek-gang-jam 756

Baek-hwa-sa 777

Baek-hwa-young-san-hong

684

Baek-jil-ryeo 644

Baem-jang-eo 763

Bae-rong-na-mu 675

Bai-bao 748

Bai-bian-dou 636

Bai-che-zhou-cao 639

Bai-du 659

Bai-fan 791

Bai-fu-zi 743

Bai-gu-ding 612

Bai-hua-shu 689

Bai-hua-you-ma-teng 638

Bai-jie 627

Baimo 733

Bai-tang-zi-shu 703

Ba-jiao 744

Ba-jiao-tou 744

Ba-jiao-ye 744

Ban-bian-lian 717

Ban-byun-ryun 717

Bandaikai 666
Banjiro 678

Bansekiryu 678

Ban-zhi-lian 709

Bareisho 711

Bark-bin-dau 636

Bark-far-yau-mar-teng 638

Bark-foo-gee 743

Bark-gie-jee 627

Bark-goo-ding 612

Bark-tou-yung 612

Bashoo-yo 744

Bashou 744

Bashou-kon 744

Bekko 772

Bel-ra-don-na 710

Beol-sa-sang-ja 681

Beradonna 710

Beradonna-kon 710

Bian-dou 636

Biao-gan-hua 736

Bi-bong 726

Bie 772

Bie-jia 772

Bi-hu 774

Bik-foo 774

Bin-dau 636

Bing-liang-hua 620

Bing-pian 626

Bing-pin 626

Bing-pyun 626

Bit-karp 772

Bo-je-su-pi 607

Bok-su-cho 620

Bong-a-chul 746

Boon-bin-lin 717

Boon-dai-hoi 666

Boon-gee-lin 709

Bor-choi 615

Borei 750

Bo-sai 615

Bosho 795

Boushou 795

Budou 662

Bung-eo 765

Buta 790

Byakkyosan 756

Byeok-o-dong 665

Byeol-bom-I-ggot 685

Byuk-ho 774

Byul-gap 772

Byung-ggot-pul 706
Calamina 797

Cang-bai-cheng-gou-feng

623

Can-huan-mao-yin 752

Can-sha 756

Cao-cong-rong 715

Catechu 632

Cham-bit-sal-na-mu 659

Cham-chui 720

Cham-gul 750

Cham-juk-na-mu 655

Cham-woe 672

Chan-chin 655

Chang-chun-hua 696

Chang-mu-li 750

Chang-po 739

Chan-su 770

Chan-tui 757-758

Charm 756

Charm-sar 756

Cha-tiao-qi 656

Cheng-gou-feng 623

Cheng-xiang 667

Cheom-gwa 672

Cheom-gwa-ja 672

Cheom-gwa-yeop 672

Cheon-geum-deung 624

Cheon-il-hong 616

Cheon-mok-hyang 730

Cheung-chun-fah 696

Cheung-poh 739

Chikujo 737

Chim-hyang-na-mu 667

Chin-cheung-gec 713

Ching-gon-chuk 737

Ching-ngau-fung 623

Chin-yut-hung 616

Chisa 729

Chi-yang 605

Cho-jong-yong 715

Chosen-matsu 603

Chu 790

Chuan-bei-mu 733

Chuan-mu-xiang 730

Chuan-por-sack 606

Chuan-po-shi 606

Chu-chong-ju 724

Chu-darm 790

Chu-eo 764

Chui-cho 649

Chuk-yue 737

Chun-back-pi 655 
Chun-gou 694

Chung-yue-sack 798

Chun-yeop 655

Chu-ye-hua-jiao 650

Ci-huai 640

Cui-lan-cao 686

Cu-jing-qin-jiao 694

$\mathrm{Cu}$-zhuang-nu-zhen 691

Da-al-ri-a 725

Da-al-ri-a-geun 725

Da-dao-lang 760

Dae-ja-seok 794

Dae-yeop-yong-dam 694

Da-fong-ai 723

Dai-huet-teng 621

Daiketsuto 621

Dak 779

Dak-pul 664

Da-lian-wan-wu-li 750

Dal-rae-ggot-mu-reut 735

Dan shu 737

Dang-geun 682

Dan-se-jin-si-yan 780

Daria 725

Da-tou-dian-zhu 737

Da-xue-teng 621

Day-ding 668

Day-lung 752

Ddae-juk-na-mu 688

Ddal-gi 629

Ddal-gi-yeop 629

Ddeok-ssuk 727

Deung-ja 648

Deung-ja-pi 648

Diang-huang-jing 734

Dian-qie 710

Dian-qie-cao 710

Di-bie 759

Die-ngai 723

Die-yip-tsau-jiu 650

Di-long 752

Ding-gong-teng 701

Dobainio 671

Doc-ji 790

Doi-jair-sack 794

Dojou 764

Dokeigai 614

Dokukakuren 743

Dol-jo-gae 749

Do-ma-baem 773

Do-ma-baem-but-I 774

Dong-feng-cai 720

Dong-pung-chae 720

Dong-pung-chae-geun 720
Dong-ya-qian-xie 754

Du-ggeo-bi 770

Duh-hung-fah 703

Du-hong-hua 703

Du-jiao-lian 743

Duo-hua-huang-jing 734

Egonoki 688

Enka 780

Enokoro-gusa 738

Er-bao 748

Er-cha 632

Er-ye-hong-shu 702

E-shu 746

Eung-su-seok 792

Fah-kay-sum 679

Fan-shi-liu 678

Fan-xie-ye 633

Farn-sack-lou 678

Farnsacklou-yip 678

Farn-sair-yip 633

Foo-ding-cha 691-692

Foo-long-shu 704

Fujimame 636

Fukahire 762

Fukuju-so 620

Gae-gam-su 646

Gae-mang-cho 726

Gag-chi-kong 636

Gainoko 723

Gaiyo 719

Gajutsu 746

Ga-mak-sa-ri 722

Gambir 632

Gam-ja 711

Gam-na-mu 687

Gam-ram 653

Gam-ram-na-mu 653

Gam-su 646

Ga-mul-chi 769

Ganbiru 632

Ga-neun-ip-geum-bul-cho

728

Gan-feng-cao 735

Gang-a-ji-pul 738

Gang-hyang-dan 634

Gang-jiu-hyang 634

Gang-song 676

Gan-lan 653

Gan-su-bei-mu 733

Gap-o-jing-eo 751

Gar-lin-kiu 705

Garm-larm 653
Gar-muet-lay 704

Garp-gie 773

Gar-wong-mar 663

Gar-ying-jao 618

Ge-jie 773

Gekkei-ju 619

Genjin 712

Genno-shouko 642

Geo-buk 771

Geo-ji-deong-gul 660

Geo-meo-ri 753

Geom-eun-baem 775

Geum-bong-hwa 700

Geum-bul-cho 728

Geum-cha-seok-gok 747

Geum-gang-a-ji-pul 738

Geum-jeon-cho 706

Geun-hwa 683

Geu-ra-di-o-ra-seu 736

Gight 779

Gight-muk 628

Gight-noi-gum 779

Gim-larn 736

Gin-byung-ggot-pul 706

Goko 755

Gol-soe-bo 602

Gomafu-azarashi 782

Gong-heung 634

Gong-tsung 676

Go-ou 785

Gotouhi 665

Gotou-shi 665

Gou-ji 606

Gou-wei-cao 738

Gou-xian-da-ji 646

Go-yom-na-mu 687

Guang-huo-xiang 707

Guang-jin-qian-cao 635

Gui-hua 693

Gui-jia 771

Guk-hwa-jwi-son-I 642

Gum-chin-bark-far-sair

776

Gum-chin-cho 635

Gu-mi-cho 738

Gum-lin-far 643

Gum-see-yin 780

Gunbai-hirugao 702

Gun-cheon-ja 687

Guo-gang-rong 637

Gu-pan 771

Gurajiorasu 736

Gu-sui-bu 602

Gwa-che 672

Gwaeng-i-bap 641 
Gwak-hyang 707

Gwang-dong-in-sam 679

Gwang-gwak-hyang 707

Gwight-barn 771

Gwong-fock-heung 707

Gwor-gong-lung 637

Gwut-shui-boh 602

Gye-nae-geum 779

Gyeo-u-sal-i 608

Gyoryuu 670

\section{Hachiku 737}

Hae-gu-sin 782

Hae-ho-ja 601

Hae-jo 601

Hae-ma 767

Hae-pyo-cho 751

Hae-ryong 768

Hae-song-ja 603

Hahakogusa 727

Hai-bao 782

Hai-gou 782

Hai-gou-shen 782

Hai-long 768

Hai-lu 685

Hai-ma 767

Hai-mang-guo 697

Hai-mang-guo-zi 697

Hai-nan-sha 745

Hai-piao-xiao 751

Hai-sheng 761

Hai-zao 601

Hakuban 791

Haku-gaishi 627

Hakuhenzu 636

Hamabishi 644

Ham-bak-i 624

Hangesho 625

Han-hao-zi 601

Han-jin-lin 643

Hannok 605

Hanpenren 717

Hanpi 777

Han-ryun 643

Han-ryun-hwa 643

Hanshiren 709

Hap-gae 773

Harabiro-kamakiri 760

Haran 732

Hari-enju 640

Hasunohakazura 624

Hei- zha 757

Hei-kuang-chan-su 770

Hei-shui-pu-tao 661

Hei-zha 758
He-lian-dou-cao 611

Henruuda 649

Hime-mukashi-yomogi

726

Hiru 753

Hitotsuba-hagi 647

Hoh 750

Hoi-gou-bin 782

Hoi-joh 601

Hoi-lung 768

Hoi-mah 767

Hoi-mong-gwor 697

Hoi-pao 782

Hoi-piu-siu 751

Hoi-sum 761

Ho-je-bi-ggot 668

Hokama-uraboshi 602

Hondawara 601

Hong-song 603

Hon-oniku 715

Ho-ra-bok 682

Hor-lin-dou-cho 611

Horutokazura 701

Hosoba-oguruma 728

Houren-cho 615

Houren-so 615

Hua-jiao 651

Huang-gua 673

Huang-jing 734

Huang-shu-kui 664

Huang-shu-kui-gen 664

Hua-shan-song 603

Hua-shi 800

Hui-mao-chuan-mu-xiang

730

Hui-yao-jin-si-yan 780

Hu-ji-sheng 608

Hu-jue 602

Huk-gay-sunk 608

Hung-wong 796

Huo-xue-dan 706

Hup-foon-far 617

Hwa-cho 651

Hwal-seok 800

Hwang-chok-gyu-geun

664

Hwang-gwa 673

Hwang-gwa-deung 673

Hwang-gwa-hwa-roe 673

Hwang-gwa-yeop 673

Hwang-hae-ssuk 719

Hwang-jeong 734

Hyang-yu-go-rae 781

Hyun-sam 712
Ibota-kusagi 704

Ichigo 629

Ichigo-no-ha 629

I-dam 766

Ikuri-nin 630

Il-il-cho 696

Il-yeop-chu 647

Indo-bodai-ju 607

In-do-bo-ri-su 607

Indo-jaboku 698

In-do-sa-mok 698

Ing-eo 766

Iou 799

I-yuk 766

Jagaimo 711

Ja-goe-hwa 640

Jah-cheung-cho 641

Jak-jang-cho 641

Ja-kou 789

Jakou-jika 789

Jam-bun 756

Ja-mi-geun 675

Ja-mi-hwa 675

Ja-mi-yeop 675

Ja-ra 772

Jashoshi 681

Jat-na-mu 603

Je-bi 780

Je-chung-guk 724

Je-don-gwa-pi 688

Jee-may 675

Jee-may-yip 675

Jeo-dam 790

Jeok-yang 605

Jeon-gal 754

Jeo-yeop-hwa-cho 650

Jeuk-eo 765

Jia-can 756

Jia-huang-ma 663

Jia-ji 779

Jia-lian-qiao 705

Jiang-can 756

Jiang-xiang 634

Jiang-xiang-tan 634

Jian-wei-yu 741

Jian-ye-fan-xie 633

Jia-ying-zhao 618

Ji-byul 759

Jicho 668

Ji-di-bie 759

Jie 627

Jie-zi 627

Ji-jeong 668

Ji-ju-po-dan 732 
Ji-li 644

Ji-mu 628

Jin-dal-rae 684

Ji-ne 755

Ji-nei-jin 779

Jin-fei-cao 728

Jingyo 694

Jin-jiang-mu-li 750

Jinko 667

Jin-qian-bai-hua-she 776

Jin-si-yan 780

Ji-reong-I 752

Ji-ryong 752

Jiryu 752

Ji-yu 765

Ji-zhao-feng 618

Jo-gyung-cho 658

Jom-cham-bit-sal-namu659

Jom-sa-ma-gui 760

Jong-yu-seok 798

Jo-pap-na-mu 631

Jo-reum-na-mul 695

Ju-fu-tang-lang 760

Juk-yeo 737

Jun-qian-zi 687

Jut-light 644

Jwi-o-ju-m-puk 716

Kagi-kurumaba-

narukoyuri 734

Kaiba 767

Kaihyoushou 751

Kaijin 761

Kaiko 756

Kaikujin 782

Kairyuu 768

Kaiso 601

Kaki 687, 750

Kakidoushi 706

Kakko 707

Kakushitsu 682

Kanran 653

Kanton-ninjin 679

Karakogi-kaede 656

Karashina 627

Karasu-zanshou 650

Kasseki 800

Katabami 641

Katei 672

Kay-sair 777

Ke-aritaso 614

Keikanseki 796

Keiketsutou 638

Keinaikin 779
Ke-teng-zi 637

Keung-charm 756

Kiban 771

Kikuba-fuuro 642

Kingyo 765

Kinmokusei 693

Kinsenhakkada 776

Kinsenso 635

Kit-cho 716

Ki-uri 673

Ko-bbul-so 784

Koi 766

Kokamakiri 760

Kossaiho 602

Kou-ika 751

Kouki-sekkoku 747

Ku kai seki 797

Ku-ding-cha 691, 692

Kui-han 749

Kukuinoki 645

Ku-lang-shu 704

Kusagame 771

Kwa-ba 678

Kyokuto-sasori 754

Lan-an 677

Lang-ba-cao 722

Lao-guan-cao 642

Lian-qian-cao 706

Lian-sheng-gui-zi-hua 700

Ling-yang-jiao 787

Ling-yeung 787

Ling-yeung-gock 787

Lin-she 789

Lin-sunk-gwai-jee 700

Liu-huang 799

Li-ye-xiu-xian-ju 631

Li-yu 766

Li-yu-dan 766

Loh-gum-sack 797

Long-lao-xiang 626

Long-yan 657

Long-yan-he 657

Long-yan-rou 657

Long-yan-xiang 781

Long-yan-ye 657

Long-zhu-guo 669

Lou-wong 799

Lu 783,788

Lu-bian 788

Lu-gan-shi 797

Lu-jiao 788

Lu-jiao-jiao 788

Lu-jiao-shuang 788

Lu-jin 788
Luk 788

Luk-bin 788

Lu-ke-sha 745

Luk-gock 788

Luk-gock-gow 788

Luk-gock-sheung 788

Luk-gun 788

Luk-mai-bar 788

Luk-toy 788

Luk-yung 788

Lung-chu-gwor 669

Lung-ngarn 657

Lung-Ngarn-Wut 657

Lung-ngarn-yuk 657

Lu-pi-jiao 783

Lu-rong 788

Lu-tai 788

Lu-wei 788

Ma-an-teng 702

Ma-dac-o-bun-ja-gi 748

Mae-mi 757

Magaki 750

Ma-huang 753

Ma-hua-qin-jiao 694

Mai-lan-cai 613

Makkou-kujira 781

Makuwa-uri 672

Ma-li-jin 700

Mal-maeng-I-na-mul 613

Mamushi 777

Manamako 761

Mang-cho 726,795

Mang-jie-huan-mao-yin

752

Mang-niu-er-miao 642

Mang-xiao 795

Man-li 763

Man-rei-gyo 763

Man-yeo-eo 763

Mao-han 749

Mar-on-teng 702

Masaki 658

Ma-sak-jul 699

Ma-she 789

Matsurika 690

Matsurikon 690

Ma-wei-song 603

Mayumi 659

Megusurinoki 656

Meo-gyi-na-mu 650

Meol-ggul 622

Mi-ggu-ra-ji 764

Mi-na-ri 683

Ming-fan 791 
Ming-farn 791

Mishimasaiko 680

Mitsuba-fuuro 642

Mitsugashiwa 695

Modama 637

Mok-hyang 730

Mokko 721

Mokukocho 713

Mo-li 690

Mo-li-gun 690

Mo-li-hua 690

Mol-yak 654

Mol-yak-na-mu 654

Mong-sui 795

Mood-lay 690

Mood-lay-gun 690

Mo-ryeo 750

Motsuyaku 654

Motsuyakuju 654

Mou-light 750

Mo-yao 654

Mo-yao-shu 654

Mu-hu-die 713

Mu-hu-die-zi 713

Muk-heung 721

Muk-woo-dip 713

Mul-gae 782

Mu-li 750

Mul-so 786

Mu-xiang 721

My-ma-teng 604

Myoban 791

Myong-ban 791

Myouban 791

Na-gung 755

Nak-seok-deung 699

Nak-seok-gwa 699

Nanbangiseru 714

Nang-pa-cho 722

Nang-pa-cho-geun 722

Nan-he-shi 682

Nan-wu-wei-zi 675

Nap-ga-sae 644

Natsutodai 646

Neok-jul-go-sa-ri 602

Ngar-ling-gee 749

Ngarp 778

Ngarp-sun 778

Ngau 785

Ngau-wong 785

Ng-boh-sair 777

Ng-gung 755

Ngie 719

Ngor-suet 746
Nichi-nichi-ka 696

Ni-han 749

Nikujuyo 715

Ninjin 682

$\mathrm{Ni}$-qiu 764

Niu 785

Niu-huang 785

Niu-nai-jiang-cao 646

Niu-teng 622

Niwatori 779

Nobudou 662

No-gam-seok 797

No-gwan-cho 642

Nojisumire 668

Nok-gak 788

Nok-hyul 788

Nok-pyun 788

Nok-sin 788

Nok-yong 788

No-yuk 783

Nozenharen 643

Nu-e 756

Nyuukou 652

Nyuukouju 652

O-dong-back-pi 665

O-gong 755

Oguruma 728

Ohfurugyo 613

Oh-Kamakiri 760

O-i 673

Oniku 715

On-sick-heung 689

Ooshokkikon 664

Oranda-ichigo 629

Or-gou 783

O-ri 778

O-ri-na-mu 605

O-ri-na-mu-deo-bu-sal-i

715

O-ryum-mae 660

O-sa 775

Osokon 686

Ottosei 782

Ousei 734

Pa-chae 615

Pa-chae-ja 615

Pa-cho 744

Pa-cho-geun 744

Pa-cho-yeop 744

Pachuri 707

Pang-da-hai 666

Pegu-asenyaku 632

Peng-e-shu 746
Po-do 662

Po-do-deung-yeop 662

Po-do-geun 662

Poe-toe 662

Pu-tao 662

Pu-tao-gen 662

Pu-tao-teng 662

Pu-ti-shu 607

Pyun-du 636

Qian-jin-teng 624

Qian-ri-hong 616

Qing-gan-zhu 737

Qing-guo 653

Qing-jiao 651

Qin-jiao 694

Qi-she 777

Quan-xie 754

Rauwolfia 698

Reigyo 769

Reiyo-kaku 787

Ri-ben-ruo-shi 699

Ritan 766

Roba 783

Rodoku 646

Rokanseki 797

Rokuben 788

Rokubi 788

Rokujou 788

Rokukaku 788

Rokukaku-kou 788

Roukanso 642

Ruo-shi 699

Ruo-shi-teng 699

Rurihakobe 685

Ru-xiang 652

Ru-xiang-shu 652

Ryugan 657

Ryugan-niku 657

Ryukyu-tsutsuji 684

Ryuunou 626

Ryuunouju 626

Ryuuzenkou 781

Sa-cheol-na-mu 658

Sack-cheung-poh 740

Sack-goh 793

Sack-kuet-ming 748

Sack-luet 645

Sa-gu-hwa 708

Sa-hyang 789

Sa-hyang-no-ru 789

Sai 784

Saiga-kamoshika 787 
Sai-jia-ling-yang 787

Saikaku 784

Saiko 680

Sa-in 745

Sair 789

Sair-chuang-gee 681

Sair-gwar 674

Sair-heung 789

Sakuraso 686

Sal-bi-a 708

Sal-mo-sa 777

Sa-ma-gui 760

Sam-back-cho 625

Same 762

Sam-so-cho 639

Sa-myun-mok 659

San-bai-cao 625

San-cho-na-mu 651

San-deung-deung-ang 661

Sang-chi 729

Sang-gi-saeng 608

Sang-piao-xiao 760

Sang-pyo-cho 760

San-mo 610

San-mo-yeop 610

Sanpakuso 625

Sansho 651

Sarm-bark-cho 625

Sarusuberi 675

Sa-sang-ja 681

Sa-seum 788

Satoimo 742

Satsuma-gokiburi 759

Seiji 708

Seiyo-shirokarashi 627

Seiyou-kanokoson 716

Sekiketsumei 748

Sekisho 740

Sekitekko 794

Sekko 793

Sekkoku 747

Senkinto 624

Sen-mokkou 730

Senna 633

Senpukuka 728

Senso 770

Senzei 758

Seo-gak 784

Seo-guk-cho 727

Seok-chang-po 740

Seok-go 793

Seok-gok 747

Scok-gyeol-myung 748

Seom-su 770

Seon 758
Seon-bok-hwa 728

Seong-ryu 670

Seon-tae 758

Seon-toe 757

Seri 683

Shachu 759

Shah-yue 762

Shah-yue-gwut 762

Shajin 745

Shakuchiri 609

Shakuchiri-soba 609

Shan-jiao 651

Shan-teng-teng-yang 661

Shao-ji-ju-wu-gong 755

Sha-ren 745

Sharien 792

Shar-yun 745

Sha-yu 762

Sha-yu-gu 762

She 789

She-chuang 681

She-gen-mu 698

She-gua 674

Sheng-liu 670

She-xiang 789

Shi 687

Shi-chang-pu 740

Shi-gao 793

Shi-hu 747

Shi-jue-ming 748

Shika 788

Shi-li 645

Shima-kuwazuimo 741

Shina-gokiburi 759

Shina-hikigaeru 770

Shinano-gaki 687

Shina-suppon 772

Shirayama-giku 720

Shiro-mushiyoke-gkiku 724

Shiro-tsume-kusa 639

Shitsurishi 644

Shkusha 745

Shobu 739

Shobukon 739

Shokusho 651

Shoku-shuyu 650

Shonyuseki 798

Shuan-mo 610

Shui-cai 695

Shui-jut 753

Shui-ngau 786

Shui-ngau-gock 786

Shui-niu 786

Shui-niu-jiao 786
Shui-qin 683

Shui-zhi 753

Shukusha 745

Shukyu 774

Shu-qu-cao 727

Siam catechu 632

Si-geum-chi 615

Sight-gock 784

Sight-hor-lau 670

Sight-ngau 784

Si-ho 680

Sim 758

Sim-dui 758

Sim-soh 770

Sim-tui 757

Sing-lau 670

Si-nian-huang 685

Sin-na-mu 656

Sin-na-mu-yeop 656

So 785

Sohyosho 760

Sokikuso 727

Sokisei 608

Som-dae 737

Song-piu-siu 760

Song-zi 603

So-yeop-hwa 631

Suan-mo 610

Su-chae 695

Su-geun 683

Sui-aen do 797

Suiba 610

Suigyuu 786

Suisaiyo 695

Suishitsu 753

Su-ji 753

Suo-sha-bei-mu 733

Su-san-hwang 736

Su-u-gak 786

Su-yeom-ga-ral-ggot 717

Su-young 610

Syamu-asenyaku 632

Taishascki 794

Takasagogiku 723

Tama-negi 731

Tamasudare 735

Tang-chang-pu 736

Taruku 800

Ta-ukogi 722

Teika-kazura 699

Teikoto 701

Tian-giao-mai 609

Tiao-ye-xuan-fu-hua 728

Tobizu-mukade 755 
To-byul-chung 759

To-ggi-pul 639

Toh-bit-chong 759

Toh-ging-gie 614

Tong-chui-yu-dai-cao 718

Tong-su-huan-mao-yin

752

To-ran 742

Tororo-aoi 664

Tou-mube 622

Touwata 700

Tsim-may-woo 741

Tsuan-bui-moh 733

Tsuan-kit 754

Tsubame 780

Tsum-Heung 667

Tu-bei-mu 671

Tu-bie-chong 759

Tu-jin-jie 614

Tung-tsui-yuk-dai-cho 718

U-hwang 785

Uk-ri-geun 630

Uk-ri-in 630

Unagi 763

Ung-hwang 796

Un-hyang-na-mu 649

Unkou 649

Ushi 785

U-sho-da 775

Usugi-mokusei 693

Wa-ban-ja 749

Wa-geo 729

Wa-geo-ja 729

Wa-leng-zi 749

Wang-bu-liu-xing 613

Wang-bul-ryu-haeng 613

Wang-meo-ru 661

Wang-sa-ma-gui 760

Wart-sack 800

Wei-lian-huan-mao-yin

752

Weol-gye-ja 619

Weol-gye-su 619

Weol-gye-yeop 619

Weol-nam-an-sik-hyang-

na-mu 689

Wi-seong-ryu 670

Wo-ju 729

Wong-gwah 673

Wong-jing 734

Won-hwang-jeong 734

Woo-chark 751

Woo-gwight 771
Woo-sau-sair 775

Wu-bu-she 777

Wu-gong 755

Wu-gui 771

Wu-li 769

Wu-lian-mei 660

Wu-pu-bi-hu 774

Wu-shao-she 775

Wu-tong 665

Wu-tong-pi 665

Wu-tong-zi 665

Wu-zei 751

Xiang-chun 655

Xiao-bai-jiu-cao 726

Xiao-dao-lang 760

Xiao-fei-peng 726

Xiao-qin-jiao 694

Xiao-ye-hua 631

Xiao-ye-mai-ma-teng 604

Xia-ye-fan-xie 633

Xie-cao 716

Xi-he-liu 670

Xi-jiao 784

Xi-jing-shi-hu 747

Xi-niu 784

Xin-se-cao 612

Xiong-huang 796

Xi-yang-shen 679

Xuan-fu-hua 728

Xuan-shen 712

Ya 778

Yabugarashi 660

Yabujirami 681

Yabutabako 683

Yadorigi 608

Ya-go 714

Yair-goo 714

Yair-hup-far 617

Yair-muk-gwar 622

Ya-mok-gwa 622

Yamokka 622

Yanbaru-hakobe 611

Yang-bao 748

Yang-chong 731

Yang-chun-sa 745

Yang-chun-sha 745

Yang-cong 731

Yang-ddal-gi 629

Yang-pa 731

Yang-seo-chae 601

Yang-u 711

Yang-xi-cai 601

Yan-wo 780
Ya-u 742

Ya-u-yeop 742

Ya-xie 778

Ya-zhou-ruo-shi 699

Ye-di-zhu 647

Ye-eo 769

Ye-giao-mai 609

Ye-gu 714

Ye-he-hua 617

Ye-huang-ma 663

Ye-hu-luo-bo 682

Ye-mo-li 688

Ye-mu-gua 622

Yeon 780

Yeon-saeng-gye-ja-hwa

700

Yeon-wa 780

Yeop-ran 732

Ye-yu 742

Yin-do-pu-ti-shu-pi 607

Ying-cao 686

Ying-hong-du-juan 684

Ying-tao 630

Yin-huan-she 776

Yi-nian-peng 726

Yin-Wor 780

Yomogi 719

Yong-an-yuk 657

Yong-jeong-hyang 781

Yong-noe-hyang-na-mu 626

Young-Yang 787

Young-yangkgak 787

Yuan-sum 712

Yu-chi 762

Yue-chee 762

Yue-gui 619

Yue-gui-zi 619

Yu-hwang 799

Yu-hyang 652

Yu-hyang-na-mu 652

Yu-ja 648

Yu-ka-ri-na-mu 677

Yukarinoki 677

Yukariyo 677

Yuk-jong-yong 715

Yu-lian 735

Yun-xiang 649

Yusura-ume 630

Yuuou 796

Yuzu 648

Zang-chang-pu 739

Zang-nu-jing 692

Za-se-bao 748 
Zendatsu 757,754

Zenzei 757

Zha-jiang-cao 641

Zhe-shi 794

Zhi-zhu-bao-dan 732

Zhong-hua-da-dhan-su

770

Zhong-hua-hu-jue 602

Zhong-ru-shi 798

Zhu 790

Zhu-dan 790

Zhu-ru 737

Zi-hua-di-ding 668

Zi-jing 675

Zi-jing-nu-zhen 691,692

Zi-jing-pi 675

Zi-wei 675

Zi-zhu 703

Zou-wen-pan-bao 748 


\section{INDEX TO DISEASES AND BIOACTIVITIES}

$\alpha$-Naphthyl butyrate esterase stimulation 615 Aarthralgia 739

Abdominal distending 618, $646,716,730,739,792$, 795

Abdominal pain 624,633 , $648,652,654,661,667$, $673,682,690,732,744$, $746,761,766,770,796$

Abnormal uterine bleeding 750,788

Abortifacient 724

Abortifacient 746

Abscess 606, 609, 650, 662, $663,674,784$

Acariasis 646

Aching 608

Acid and alkaline phosphatase inhibition 731

Acroesthesia 672

Acroparalysis 647

Acute and chronic bronchitis 616

Acute appendicitis 621

Acute conjunctivitis 615, 662

Acute gastritis 607, 629, 648, 672, 722, 726

Acute hepatitis 611

Acute pharyngitis 785

Acute respiratory infection 604

Aflatoxin production inhibition 731

Agglutinating 682

Aggregation of platelet 608

Alcohol poisoning 648

Alcoholism 672, 673

Aldose reductase inhibition 706,747

Allergenic 615, 619, 648, 724

Alopecia 672

Alopecia areata 602

Amenia 722

Amenorrhea 613, 621, 628, $638,645,675,693,732$, $746,753,759,772,789$

Amine tetrazolium reductase inhibition 615
Aminonucleoside nephrosis prevention 680

Amnesia 759

Amylase release stimulation 615

Anaemia 662, 788

Anaesthesia 770

Analgesic 655, 666, 669, $670,677,680,689,701$, $746,754,762,775,789$, 790

Anaphylaxis antagonist 706

Anasarca 717

Ancylostomiasis 614, 648

Aneilema 720

Anemia 615, 629, 638, 648, 657, 729, 783

Anesthetic 651

Angina pectoris 634

Angioprotective 662

Angiotensin-converting

enzyme inhibition 662

Anorexia 648, 721, 731, 734, 745, 747, 765, 778

Antagonistic 671, 702, 770, 793

Anthelmintic 614, 796

Anthrax 739

Anti-aging 768, 788

Antiaging effects 715

Antiallergenic 681, 731, 758

Antiallergic interleukin 4

formation inhibitor 677

Antiamebic 607, 708, 746

Anti-arrhythmia 608, 620, 662, 679, 698

Antiasthmatic 604, 644, $681,719,731,773$

Antiatherosclerosis 602

Antibacterial 607, 619, 626, $628,633,636,641,645$, $656,662,676,677,678$, $682,696,708,711,712$, $719,721,725,737,740$, $746,752,764$

Anti-barium 745

Antibiotic 643

Antibiotic resistance

inhibition 720

Antibotulism 601

Anti-cancer 673, 719
Anticarcinogenesis 731

Anticholinergics 710

Anticlastogenic 731

Anticoagulant 731, 753 761, 777, 789

Anticomplement 680,746, 788

Anticonvulsant 740, 754, 755, 756, 758, 785, 790

Anticrustacean 607

Anticulsive 682

Antidepressantfor

depression 766

Antidiabetic 677, 678

Antidiarrheal 726

Antidiuretic 696

Antidysenteric 682

Antiedema 615, 708, 731, 728

Antifatigue 772

Antifeedant 643, 704

Antifertility 639, 682, 753

Antifilarial 731

Antifungal 601, 607, 610, $629,640,662,685,696$, $700,707,708,711,726$, $729,731,732,742,746$, $754,755,761,782$

Antigoitrogenic 601

Anti-hepatitis 673

Antihepatotoxic 711, 746

Antihistamine 678, 709, 745

Anti-HIV 668

Antihypercholesterolemic

662, 696, 746

Antihyperglycemic 607 , 673, 677, 696, 731

Antihyperlipemic 696, 731

Anti-hypertension 608, 625, $644,666,679,712,717$, $721,726,752,777$

Antihypertriglyceridemia

662

Antiimplantation 746

Anti-inflammation 606 , $609,610,621,637,639$, $646,656,670,676,677$, $678,680,689,696,701$, $702,704,706,708,731$, $746,762,770,773,775$, $785,786,788,789,790$ 
Antileukemia 608, 611

Antimalarial 614, 615, 705, 722

Antimicrobial 654, 656, 677, 750, 785, 796

Antimitotic 696

Anti-mutation 615, 648, 662, 696, 708, 711, 731, $746,762,771$

Antimycobacterial 708

Antimycotic 681, 719

Antimycotic 719

Anti-myocardial 679

Antineoplastic 601, 671, 728

Antinociceptive 645, 678, 702

Antiobesity 665

Antioxidant 615, 630, 632, $651,661,662,677,679$, $684,706,708,711,715$, $723,767,773,785,788$

Anti-platelet agglegation 638,680

Antipyretic 680, 694, 708, $758,784,785,786,787$, 793

Antiscolic 651

Antiseptic 689

Anti-shock 679, 770

Antisickling 731

Anti-skin aging 605

Antispasmodic 702, 706, 721, 724, 740, 744, 746, 785

Antispermatogenic 696

Anti-SRS-A 719

Anti-stress 767, 773, 788

Antithermic 712, 719

Antithrombotic 731, 761, 767,782

Antitrichomonal 681

Antitrypsin

and

hemagglutinating 636

Antitumor 608, 609, 615, $624,633,634,643,696$, $677,714,731,744,746$, $749,752,755,756,761$, $768,770,782$

Antitussive $609,611,644$, $677,681,719,723,733$

Antiulcer 639, 680, 706, 722,744
Antiulcerogenic 619

Antivenomous 717

Antiviral 607, 633, 636, $643,662,666,671,680$, $687,696,704,706,708,711$ Antiyeast 708, 711, 731, 746

Anxiety 695, 750

Aorta calcium antagonist 679

Apoplectic hemiplegia 754, 755, 776

Apoplexy 626, 673, 680, 778

Appendicitis 709, 717, 795

Arrhythmia 792

Arrythmias 683

Arteriosclerosis 658, 731

Arthralgia 752, 769

Arthritis 602, 684, 694

Articular pain 677, 699

Ascariasis 614, 630, 650, 651, 682, 687, 724, 746

Ascites 611, 612, 625, 646, 709, 717

Ascites hepatoma cells 709

Asthma 607, 667, 684, 727, 752, 766, 773, 781, 790, 793, 798, 799

Ataxia 647

Atherogenesis 762

Atrial fibrillation 698

Axillary abscess 622

Azoalbumin hydrolytic 77

Azocaseinolytic 777

\section{Back pain 788}

Bacteriocide 799

Bacteriostasis 625, 651, $668,681,707,709,717$, 728,656

Bad breath 632

Barbiturate sleeping time decreasing 746

B-cell proliferation 680

Bee sting 731, 741, 742

Belching 667

Beriberi 608, 624, 625, 630,

$637,673,744,763,769$

Bile secretion 785

Binding of aluminium ion with proteins 791

Bleeding 634, 655, 791, 794
Bloodshot eyes 662,738

Blood pressure depression 698

Blood stasis 654,753

Blurred vision 616,748

Boil 606, 625, 641, 663, $668,674,675,702,705$, $709,711,712,723,736$, $764,765,767,768,770$, 788, 791, 796, 799

Bone fracture 602,759

Brain mitochondria 754

Breast cancer 764

Bronchial asthma 616

Bronchitis 604, 684, 686, 700, 703, 713, 722, 727

Bronchodilator 731

Bronchospasm 681

Bruise 652, 678, 746, 759, 764

Burn 628, 641, 650, 673, $677,703,711,742,744$, 764,793

\section{Cacanthrax 796}

Caccaogue 666

Calcaneal abscess 705

Calcium antagonist 707

Calcium influx 767

Calmative 743

Cancer 659, 762

Carboxylesterase 682

Carbuncle 611, 612, 624, $643,652,654,664,668$, $669,683,700,717,723$, $729,731,742,744,767$, $770,784,785,787,788$, 789,796

Cardiac 608

Cardiac disease 620

Cardiac disorder 789

Cardiac edema 607

Cardiac infarction 621

Cardiac infuction 785

Cardiac insufficiency 770

Cardiac palpitation 657

Cardiac weakness 679

Cardialgia 781

Cardiopalmus 695

Cardiorelaxant 641

Cardiotonic 604, 644, 663, 700, 786

Cardiotoxic 724 
Cardiovascular 628,679 , 701,703

Cardiovascular stimulating 789

Caseinolytic 777

Catalase stimulation 615

Caustic soda poisoning 778

Cellulitis 741

Centipede bite 742

Central analgesia 793

Central depressant 680

Central M-cholinergic 701

Cerebral apoplexy 698

Cerebral hemorrhage 744

Cerebral hyperemia 744

Cervical cancer 746

Cessation of the milk secretion 798

Chest distention 680

Chin cough 616

Cholagogue 635

Cholecystitis 623, 695, 726

Choleretic 745, 746

Cholinergic mechanisms 776

Cholinesterase 776

Cholinoreceptive

membranes 776

Chronic arthritis 628, 754

Chronic bronchitis 601,670

Chronic constipation 633

Chronic diarrhea 791

Chronic dysentery 636, 682

Chronic enteritis 678

Chronic lymphadenitis 671

Chronic malaria 772

Chronic nephritis 636

Chronic rheumatic 755, 777

Chronic rheumatiod arthritis

776

Chronic sores 799

Chronic ulceration 788

Chyluria 657

Cirrhosis 709, 785

Clastogenic 662

CNS 770

CNS depressant 678, 708

Coagulant 731

Coiled incens stick for

fumigation 724

Cold 628, 641, 648, 650, $651,670,684,690,704$, $707,720,743$
Colistaxis 640

Collagenase inhibition 662

Coma 781, 785

Common cold 624, 631, $677,683,702,719,723$, $727,731,738,757$

Common cold relief 662

Complement 776

Congestive heart failure 620

Conjuctival congestion 720

Conjunctivitis 643,660 , $669,690,712,726,758$, $787,790,791,797$

Connective tissue proliferation 772

Constipation 603, 611, 615, $633,642,662,664,666$, $672,674,697,712,715$, $790,792,795,799$

Consumptive fever 771,772

Contractile 666

Contraction of mouse small intestine 723

Contracture 652, 777

Contusion 642, 684, 699, 711, 720, 732

Convulsion $649,752,757$, $775,776,777,784,785$, 786, 787, 788

Cordial 770

Corn 629

Corticosterone secretioninducing 680

Corticotropin 680

Costal pain 617

Cough 603, 617, 640, 653, $669,674,679,682,684$, $686,693,706,713,723$, $727,728,732,737,739$, $742,752,757,758,766$, $773,780,781,783,793$, 798

Cough with bloody sputum 733

Cough with sputum 674

Cutaneous pruritus 764

Cyclooxygenase inhibition 731

Cystitis 713, 715

Cystolithiasis 706

Cytotoxic 607, 610, 631, $639,649,659,676,681$, $688,696,699,700,706$,
$709,711,726,731,746$

Deafness 647

Debility 761, 762, 771, 778, 780, 788, 789

Decreasing bile output 793

Decurative 717

Decurtating blood

coagulation time 793

Deep-rooted boil 655

Deficiency 602, 734

Deficient milk after childbirth 662

Deficient of milk 729

Delirium 739, 785, 786, 787

Depigmentation 662

Deprementia 781

Depression 745

Depression of respiration 777

Dermatitis 702, 722, 723, 727,738

Dermatomyces 614,727

Dermatopathy 610, 738

Detoxication 778

Deviation 743

Diabetes 607, 615, 678, $679,687,696,706,725$, $727,734,744,756,764$, 765 ,

Diarrhea 605, 609, 624, $628,629,632,642,649$, $651,660,673,677,678$, $683,690,717,721,723$, $730,739,742,770,799$, 800

Diastolizing 752

Difficult delivery 767

Difficult in urination 610 , 630, 662

Dilating coronary artery 682

Dimethylcaseinolytic 777

Diphtheria 712, 739

Dire thirst 653

Disease after child-birth 769

Distending pain 644,721

Diuretic 660, 708, 731

Dizziness 603, 616, 644, 720, 748, 750, 772, 787

Dolor 710

Drastic irritation 614

Dry cough 666, 733, 734

Duodenal ulcer 622 
Dysentery 607, 610, 612, $616,641,642,653,655$, $660,663,668,673,675$, $678,684,707,717,722$, $726,739,740,744$

Dysfunctional uterine bleeding 783

Dysgalactia 664

Dysgalactia 729

Dysmenorrhea 608, 609, $613,618,621,622,658$, $675,706,723,746,769$

Dyspepsia 651，655，745, 746

Dyspnea 767, 780, 794

Dystocia 789

Dysuria 622, 625, 670, 672, $694,726,729$

Earache 603

Ecchymosis 788

Ecthyma 678

Eczema 603, 614, 619, 624, $625,632,649,651,675$, $676,681,700,704,722$, $723,756,791,793,797$, 800

Edema 601, 620, 622, 624, $630,633,637,646,660$, $662,664,669,672,673$, $683,686,706,709,717$, $721,725,732,744,752$, $764,765,766,778,792$, 795, 800

Edema with oliguria 635

Effect against anoxia 759

Effect on rabbit electrocorticogram 701

Effect on womb 752

Elastase inhibition 615,662 , 711

Emaciation 655, 788

Embryotoxic 639

Emesis 648

Emetic 672, 721

Endometrorrhagia 628, 703

Enhance the effect of cosmetics on the skin 602

Enhancing effect on tolerance against hypoxia 790

Enteritis 612, 641, 642, 653, $655,657,676,677,678$,
$717,720,722,726,760$, 767,779

Epidemic fibrile diseases 787

Epidermal growth factorlike 780

Epigastric pain 611, 634, 713, 750

Epigastrium 721

Epilepsy 620, 626, 639, $673,698,735,739,740$, $759,784,785,786,787$, 788, 789, 791, 792, 794

Epistaxis 603, 605, 615, $628,641,659,703,719$, 784

Erysipelas 660, 668, 684, 722, 726, 784

Erythrocyte adhesion 751

Erythrocytic membrane $\mathrm{Na}, \mathrm{K}$-ATP enzyme 754

Esterase 755

Estrogenic 607, 639, 681, $682,708,711,761,773,782$

Ethanol absorption

inhibition 619

Excessive leukorrhea 681, 750, 791

Excessive menstrual flow 719

Exciting effect on the nerve and skeletal muscle 754

Expectorant 616, 644, 689, 709, 728, 778

Extrasystole 698

Exudation 797

Eye convulsion 656

Eye diseases 626, 656, 766, 796, 797

Facial maxillary inflammation 785

Facial paralysis 647, 756, 776

Fainting 772

Favism 727

Favus 684, 799

Febrile diseases 626, 679, $691,692,712,758,793$

Feebleness 624

Feeling of coldness and pain 798

Female's gonorrhea 687
Festered scrofula 776

Festering wound 632

Fever 631, 641, 649, 663, $672,676,680,687,690$, $694,698,704,707,744$, $772,778,784,786,787$, 788

Fever in infants 694

Fibrinogenolytic 777

Fibrinolysinic 752

Fibrinolytic 759, 761, 777, 782

Fidgetness 743,800

Fish or crab poisoning 653

Flaccidity 604

Flooding 675, 765

Food 764

Forgetfulness 657, 740, 771

Formation of mass 759

Fracture 602, 606, 611, 662, 700

Frequent urination 760

Frigidity 681

Frost-bite 610, 630

Fungicide 649, 694, 799

Furuncle 660, 722, 731, $736,738,785$

Furunculosis 714

Furunculus 768

Galactagogue 603

Galactostasis 608, 613

Gall stone 706

Ganglionic transmission 776

Gastoroenteritis 614

Gastric and duodenal ulcer 606,628

Gastric antisecretory 639, 746

Gastric pain 618, 637, 657, 749, 798

Gastric secretory inhibition 746

Gastric ulcer 622, 711, 751

Gastritis 693, 695, 704, 794

Gastroatony 679

Gastroduodenal ulcer 706

Gastroenteritis 654, 708, 731

Gastropathy 615

Gastrospasm 683

General weakness 715 
Giant follicular lymphoma 696

Giddiness 662

Gingivostomatitis 726

Glucose metabolism stimulation 615

Glucuronidase inhibition 662

Glycoprotein Ib antagonistic 777

Goiter 601, 749

Gonadotropin release

stimulation 615

Gonalgia 788

Gonorrhea 610, 640, 641, $664,683,781$

GOT inhibition 746

GPT inhibition 731, 746

Grey hair 692

Guanylate cyclase 776

Haematemesis $660, \quad 703$, 783

Haemoptysis of pulmonary

tuberculosis 616

Haemorrhagia 786

Hageman factor inhibition 711

Hair growth stimulant 739

Headache 616, 644, 680, $684,690,694,720,732$, $748,754,756,786,787$, 793

Heat stroke 663

Hemafecia 655

Hemafecia 783

Hemagglutination 615, 640, 711,763

Hematemesis $610,634,640$, $641,643,675,684,699$, $706,765,784$

Hematochezia 605, 615, 791

Hematopoietic 788

Hematorrhea 751, 784

Hematuria 660, 729, 783, 788

Hemipelgia $647,701,752$, $756,774,775,777$

Hemodynamics 679

Hemolytic 776

Hemolyzative 755

Hemopoiesis 785

Hemoptysis 606, 640, 675,
$732,773,778,780,783$, 784,798

Hemorrhage 607, 618, 683, $699,755,777$

Hemorrhoid 639, 641, 665, $675,694,764,769,777$, 799

Hemorrhoidal bleeding 702, 763

Hemorrhoidal pain 637

Hemorrhoids 615, 659

Hemostasis 628, 703, 761, 782

Hepatic protein synthesis stimulating 680

Hepatitis 609, 641, 672, $680,704,709,722,738$, 785

Hepatoma inhibitor 715

Hepatoprotective 606, 653, $670,676,714$

Hepatosplenomegaly 606, 704, 746

Hernial pain 637,657

Hiccup 667,794

Hide powder azure

hydrolytic 777

High fever 752

Histamine release inhibition 747

Hmorrhage 703

Hoarseness 666, 713

Hyaluronidase inhibition 662

Hypercholesterolemia 731

Hyperemesis gravidarun 737

Hyperplastic rhinitis 791

Hypersensitive diarrhea 726

Hypertension 615, 641, 673, $679,695,696,698,723$, $727,744,752,754$

Hypertrophic rhinitis 671

Hypnotic 740

Hypocholesteremic 761, 782

Hypocholesteremic 782

Hypocholesterolaemic 603, 720

Hypogalactia 757,766

Hypoglycemic 610, 615, $678,711,712$

Hypolipidemic 603, 749

Hypolycemic 749
Hypotensive 708, 731, 785, 786

Hypothermal 740, 680, 739

Hypotonia 693

Hypoxia tolerant 621

Hysteria 649, 719

Hysterorrhea 706

Icteric hepatitis 606,676 , 706

Immunity 636

Immunomodulatory 714 , $751,762,773,785$

Immunoregulatory 680

Immunostimulant 609, 679, $761,772,789$

Immunosuppressant 671 , 731

Immunosuppressive 758

Impaired consciousness in heat-stroke 770

Impaired hearing 740

Impairment

of

consciousness 785

Impairment of speech 743

Impotence $647,681,715$, $747,761,764,767,768$, $773,782,788,789$

Impotence with cold lower extremities 799

Improving leukocyte and radiation-resistant 770

Improving urinary output 793

Inability to conceive 788

Incised wound of hand 726

Increasing effect on intraventricular pressure and $\mathrm{dp} / \mathrm{dt} 754$

Indigestion $612,618,649$, $673,695,739,746,779$

Induced differentiation

effect on HL-60 671

Infant astonishment 757

Infant cough 675

Infantile aphtha 665

Infantile convulsion 689 , $735,754,755,756,789$, 796

Infantile epilepsy 757,758

Infantile fever 616

Infantile indigestion 678

Infantile malnutrition 779 
Infantile measles 769

Infection 691, 692, 703

Infectious hepatitis 713,726

Infertility 719

Inflammation $736,742,757$, 797,800

Influenza 657, 706, 741

Inhibiting rhinoviruses 770

Inhibition of tumor promoter 723

Inhibitory activity on platelet aggregation 651

Inhibitory effet on prostaglandin biosynthesis 634

Inhibitory effect on acetic acid-induced writhing 789

Inhibitory effect on aggregation of platelets 707

Inhibitory effect on angiotensin-converting enzyme 643

Inhibitory effect on blood coagulation 601

Inhibitory effect on CNS $634,733,740$

Inhibitory effect on platelet aggregation 719

Inhibitory effect on prostaglandin biosynthesis 638,702

Inhibitory effect on RNA biosynthesis 761

Inhibitory effect on spontaneous motility in isolated ileum 789

Inhibitory effect on uterine contraction 682

Insect attractant 711

Insect bite $612,641,675$, $677,705,717$

Insect growth inhibitory 704 Insect repellant 693,724

Insecticide 705, 708, 724, 726,799

Insomnia $617,657,662$, $690,693,695,716,723$, $729,731,737,750,783$

Insulin receptor binding 615 Insulin sectetion-stimulating 750

Intestinal absorption inhibition 746
Intestinal motility inhibition 746

Intractable phlegm 688

Intraocular

pressure

reduction 615

Irregular mense 675

Irregular menstruation 622, $638,675,700,706,718$

Irritability 785

Itching $614,719,751$

Jaundice $611,625,637,641$, $658,660,668,672,673$, $683,694,695,709,717$, $720,736,744,764,765$, $766,790,800$

Jaundice with dark urine 635

Juvenile hormone 662

Kallikrein inhibition 711

Killing hookworm 682

Lactation 765

Lapactic 633

Large intestine bleeding 640

Larvicidal 696

Laryngitis 708, 756

Laxative 633

Leokorrhea 763

Leprosy 777

Leptospirosis 741

Leucopenia 638

Leucorrhea 609, 617, 641, $657,675,687,718,751$, 788

Leukemia 696

Leukemic hemocyte 709

Leukopenic 696

Lipase release stimulation 615

Lipid lowering 673

Lipid peoxide fromation inhibition 662

Lipid peroxidation 606

Lipid peroxide antioxidant 678

Lipoprotein lipase

stimulation 662

Lipoxygenase inhibition activity 731

Lithargiuria 641

Liver cancer 774

Liver protective 789

Local anesthesia 770
Lochia 788

Loss of consciousness 689

Lumbago 634, 638, 647, $648,659,661,662,681$, $693,704,715,727,732$, $739,747,761,766,782$, 783,788

Lumbar muscle strain 702

Lumbricidal 740

Lung abscess 709

Lung-fever 603

Lymph node tuberculosis 774

Lymphadenitis $755 ， \quad 789$, 736

Lymphnoditis 669

Lymphocyte proliferation stimulation 662

Lymphocyte stimulation 679

Lymphoid tuberculosis 609

Lymphosarcoma 696

Macular eruptions 784

Mad dog bite 685

Malaria 611, 631, 680, 704, 705, 707, 720, 796

Maldigestion 672, 673

Malignant lymphatic tumors 696

Malignant ulcers 799

Mall formation in the abdomen 750

Malnutrition 655, 682

Mania 785,791

Mass 749, 772, 789

Mass formation 753

Mass in abdomen 767, 768

Mastitis 613, 644, 671, 673, $700,709,756,788,795$

Material of atropine sulfate 710

Measles $630,662,670,757$, 758

Melancholia 783

Melena 628, 675

Membrane

fluidity

decreasing 680

Membranous fluidity 754

Menolipsis 649

Menorrhagia 657, 794

Menorrhagia 794

Menorrhalgia 658 
Menoxenia $649,654,665$, 753

Menstrual disorders 680 , 719

Mental disease 672

Meteorism 610

Metrorrhagia 683, 744, 788, 794

\section{Metrostaxis 744}

Migraine 743, 754

Miliaria 800

Miosis and decrease the intraocular pressure 701

Mitogenic 746

Mitogenic response 780

Modulation of brainstem

GABAergic 679

Molluscicidal 637

Morbid leukorrhea 625

Mouse isolated intestine contraction 677

Mumps 660

Muscula contracture 776

Muscular excesive exciting 793

Mutagenic 662

Myalgia 699, 732, 764

Myiasis 614

Myocardial ischemia 621

Myocardium 770

$\mathrm{Na}+, \mathrm{K}+$-ATPase 770

Nasal polyp 791

Nausea 743, 794

Nebula 644

Nematocidal 708

Neoplasm 709

Nephritic edema 766

Nephritis 611, 673, 680, 727

Nerval pain 677

Nervous headache 661

Neural headache 622

Neuralgia 631, 648, 684, 694,742

Neurasthenia 647, 657, 729, 731

Neuroleptic 607

Neuromuscular blocking 746

Neuronal acetylcholine sensitivity 776

Neuronal nieotinic receptor antogonic 776
Neurosism 641, 716

Neurotoxic 776

Neutralizing 702

Night blindness 748

Night screaming 616

Night sweat $609,662,722$,

$747,750,771$

Nocturnal emission 718,

$760,761,782$

Nodulations 788

Noma 765

Nucleotidase inhibition 731

Numbness of extremities 637

Numbness of the limbs 752

Obstipation 661

Oligogalactica 766

Oliguria 752

Ophthalmia 629

Opium poisoning 778

Optic atrophy 748

Ostalgia 699, 732

Osteoblast precursors 788

Osteomyelitis 714

Osteoporosis 681

Otitis media 726, 744, 791

Oviposition stimulating 700

Ovulation inhibition 639

Oxyuriasis 614,682

Pain 652, 689, 701, 716, $719,730,781,789$

Pain and swelling of the throat 653

Painful swollen

hemorrhoids 795

Palpitation 620, 662, 737, 750, 778

Panaris 742

Pancreatic secretion

stimulation 615

Pancreatitis 604

Papaverine 682

Paralysis $619,672,775,740$

Parasitosis 651, 682

Parasympathomimetic 701

Parotiditis 664, 711, 736

Passive cutaneous

anaphylaxis depression 745

Perfume 652

Peritonitis 744

Pernicious vomiting of pregnancy 745

Pertusis 713

Pharyngitis 632, 653, 686, 699, 714, 795

Pharynx 795

Pheromone 656

Phospholipase 755

Phototoxicity 725

Phthisis 712

Piejunctional adrenergic 754

Pituitary-adrenocortical axis stimulating 680

Plama cholesterol-lowering 680

Plant growth inhibition 724

Plant root growth stimulation 746

Plaque formation

suppressant 607

Plasma protein 772

Plasmic

embrane

permeability 761,782

Platelet activiation factor inhibition 706

Platelet adhesion inhibition

\section{1}

Platelet aggregation 755 , 761, 777, 785

Platelet aggregation inhibition 662, 731

Platelet-activating factor (PAF) 761, 782

Pleurisy 629

Pneumonia 700, 703

Poisoning 648, 784, 787

Poisoning by meat 711

Poisoning with alcohol 673

Poliomyelitis 650

Pollution 655

Positive inotropic 697, 700, 785

Postoperative pain 622,661

Postpartum abdominal pain 618

Postpartum bone pain 723

Potent inhibitory effect on inflammatory ear edema 671

Potentiation of $\beta$-adrenergic action in isolated guinea pig trachea 789

Preneoplastic lesion 697 
Prickly heat 673, 729

Proliferation chondrocytes 788

Promotion of gastric excretion 779

Prostaglandin-like 788

Prostration 789

Protease inhibition 696

Protease or elastase inhibitor 645

Protease(HIV) inhibition

607

Protection of low density lipoproteins 679

Protective effect against poisonous snake venom 741

Protective effect on liver injury 679

Protein synthesis inhibition 615

Proteolytic 615, 755

Pruritus 670, 676, 738, 756, 758, 797

Psoriasis 726, 799

Psychataxia 639

Psychoneurosis 766

Pterygium 611

Pulmonary metastasis 762

Pulmonary tuberculosis 741, 763

Pyodermas 675, 702, 705, 723

Pyogenic infections 728

Quenching thirst 793

Quinone recuctase induction 615

Rabbit sinoatrial node electrophysiological 679

Radiation-protective 751

Radical scavenging 662

Rectal prolapsus 641

Regurgitation 765

Relaxant activity on isolated rabbit ileum 641

Release of noradrenaline 754

Renal stones 635

Renal toxic 611

Respiratory and circulatory systems 793

Respiratory stimulant 717
Respiratory-enzyme 776

Restlessness 737

Restlessness 743

Retention of sticky phlegm difficult to expectorate 749

Reumatalgia 649

Rheumarthritis 659

Rheumatalgia 660,661 , 662, 699, 726, 732, 756, 788, 789

Rheumatic arthlargia 604, $608,618,621,622,657$, $665,670,683,701,702$

Rheumatic arthritis 624, $637,650,675,694,704$, $706,720,723,739$

Rheumatic or rheumatoid arthlalgia 642

Rheumatic pain 606,718

Rheumatism 619, 634, 648, $676,693,734,762,775$

Rheumatoid arthritis 603, $755,774,777$

Rheumatic arthritis 623

Ribosome-inactivating 674, 704

Ringworm 610

Ringworm infection 704

RNA biosynthesis 782

RNA polymerase 788

\section{Salivation 764}

Sarcoma 180709

Scabies $610,615,619,641$, $650,655,669,675,684$, $724,769,775,791,796$, 799

Scabies ringworm 777

Scald 676, 650, 793

Schizophrenia 698, 766

Sciatica 622, 661, 704

Scorbutus 615

Scrofula $601,683,712,743$,

$749,750,754,768,774$

Scrotal eczema 657

Sebaceous secretion stimulation 708

Sedative $657,680,682,697$, 716, 739, 740, 756, 758, $775,785,787$

Seizures 754

Seminal emission 750,773 , 788
Senile asthma 780

Senile infirmity 783

Significantly decreasing platelet aggregation rate 679 Significantly facilitates male copulatory behaviors 679

Single calcium channel blocking 679

Sinusitis 673

Sinusitis with headache 707

Skin ulceration 797

Skin-palsy often accompanying pruritus 673 Slow reacting substance of anaphylaxis antagonist 708

Smooth muscle 770

Smooth muscle relaxant 708,746

Snail malformation 619

Snake bite 611, 612, 623, $624,630,650,660,668$, $675,676,685,698,709$, $714,717,720,727,729$, $741,755,796$

Soar throat 758

Sodium channel blocking 754

Somnic 698

Sore 625, 664, 666, 709, 712, 754, 755, 761, 787, $788,791,800$

Sore throat $609,624,626$, $631,632,660,663,675$, $688,712,713,720,722$, $736,743,756,770,786$, 789

Spasm 754

Spasmolytic 649, 682, 688

Spastic paralysis 642

Speramatorrhea 779,782

Sperm agglutination 711

Spermatorrhea 751, 760, $761,768,788$

Spermicidal 671, 752

Spitting of blood 719

Spontaneous sweating 750

Sputum 739, 756

Squalene biosynthesis 761, 782

Stiff tongue vomiting 737

Stimulation of central nervous system 626 
Stimulation of cytokine production 679

Stomach ache 609, 624, $653,661,665,676,695$, $739,744,745,765$

Stomach ulcer 751

Stomatitis 632, 665

Strain 604

Stroke $737,743,774,785$, 789

Stuffiness sensation in the epigastrium with anorexea 740

Styptic 633

Subcutaneous infections 788

Subcutaneous ulcer 655

Summer-damp affection 707

Superoxide dismutase-like 636

Suppuration $641,677,711$, 733

Surgical ointment 697

Swellfish poisoning 619

Swelling 709, 729, 731, 751,769

Swelling and pain of the testis 601

Swollen testes 622

Sympathomimetic 731

Syncope 626, 689, 789

Syphilis 672,688

Tachysystole 750,770

Taeniasis 682

Taenicidal 746

Tenesmus in dysentery 721

Teratogenic 711

Testosterone-like 768, 773, 789

Tetanus $743,754,755,757$, 776,777

The lethal and growthinhibiting effects on Eca 109 cells 754

Therapeutic 671

Thiamin-binding 636

Thioredoxin-linked

reductive 776

Thirst 800

Threatened abortion 608, $662,737,745$

Thrombin-like 777
Thromboxane B-2 synthesis inhibition 662, 731

Tidal fever 780

Tinea 796, 799

Tinea pedis 676

Tinnitus 739, 750, 794

Tonify blood 783

Tonsillitis 653, 664, 673, $699,700,703,714,717$, $722,727,729,756$

Tooth ache 605, 607, 624, $626,649,675,688,693$, $711,726,729,732,756,765$

Tooth caries 603

Toothache Ulcers difficult to heal up 793

Topoisomerase I inhibition 642

Total erythrocyte membrane ATPase 756

Toxic effect to house fly 704

Toxicity $621,666,701,717$, 742,770

Trachycardia 698

Traumatic bleeding 710, 751

Traumatic chest pain 705

Traumatic ecchymosis 705

Traumatic fractures 650

Traumatic hemorrhage 628 , 645,703

Traumatic injuries 602, 604, $605,606,617,618,622$, $624,628,632,634,637$, $641,643,649,650,652$, $654,656,660,665,675$, $690,704,706,709,716$, $723,727,728,732,736$, $742,744,753,767,768$, 789,797

Traumatic keratitis 669

Traumatic pain 661

Traumatic swelling 701

Traumatic swelling and pain 621

Trembles 710

Tremor 771, 772

Trichomonas vaginitis 731

Trigeminal neuralgia 622

Tripterygium wilfordii poisoning 706

Trypsin inhibition 711
Tuberculosis $606,722,734$, $738,773,774,788$

Tuberculosis of cervical lymph nodes 671

Tumor 727, 742

Tumor angiogenesis 762

Tumor Necrosis Factorinducing 751

Turbid discharge with urine 760

Tussis $662,672,706,778$

Twitching 754

Tympanitis 626,791

Typhoid fever 741

Tyrosinase inhibition 662

Ulcer $626,632,717,731$, 785

Ulcer bleeding 604

Ulticaria 757

Upper respiratory infection 757

Urethritis 623，641，700, 706,714

Uretic 644, 666, 717

Urinary crystal 644

Urinary infection 655,722 , 800

Urinary tract infection 683 , 744

Urination 764

Urination difficulty 676

Urination disturbance 635

Urine difficulty 624,673 , 738

Urolithiasis 800

Urticaria 644, 670

Uterine bleeding 655,675 , 719

Uterine stimulant 688

Uterotonic 613, 761, 771, 782

Vasculitis 659

Vasodilative 621, 782

Venomous snakebite 743

Vertigo 647, 758, 771, 788, 794

Vesication 672

Viricidal 681

Vitiligo 602

Vomiting 629, 651, 667, $673,707,730,737,765$, 
Vomiting diarrhea 636

Vulnus 731

\section{Wart 720}

Weakness 603, 608, 615, $629,636,644,679,688$, $734,747,763,765,769$, $771,777,783,798$

White dysentery of child 778

White hair 665

Whooping cough 616,675 , 742,790

Wind-dampness syndrome 656

Wound 686, 751

Wound hemorrhage 675

Xanthine oxidase inhibition 662

Yin-deficiency of spleen and kidney 788 